\title{
The GRAPPA Sonographic Enthesitis Workshop
}

\author{
Lihi Eder, Gurjit S. Kaeley, and Sibel Zehra Aydin
}

\begin{abstract}
Enthesitis is a key feature in psoriatic arthritis (PsA) and may be the initial site of musculoskeletal inflammation in patients with PsA. Ultrasound (US) could improve the accuracy of clinical enthesitis assessment, but at present no consensus exists on a global sonographic enthesitis scoring method that can evaluate the extent of enthesitis at the patient level. The Group for Research and Assessment of Psoriasis and Psoriatic Arthritis (GRAPPA) US Working Group has set up a goal to optimize the evaluation of enthesitis in patients with PsA using US through the development and validation of new instruments using a combined data-driven and expert opinion-driven approach. This article summarizes the GRAPPA US Working Group's recent activities and focuses on a 2-day workshop that the group held following the annual 2018 GRAPPA meeting in Toronto, Ontario, Canada. (J Rheumatol Suppl. 2019 June;95:51-3; doi:10.3899/jrheum.190117)
\end{abstract}

Key Indexing Terms:

\section{PSORIASIS PSORIATIC ARTHRITIS}

GRAPPA
ULTRASOUND
Enthesitis is a key feature in psoriatic arthritis (PsA) and may be the initial site of musculoskeletal (MSK) inflammation in patients with PsA ${ }^{1}$. Therefore, the assessment of enthesitis has been recommended in every patient with $\mathrm{PsA}^{2}$. Ultrasound (US) could improve the accuracy of the clinical assessment of enthesitis ${ }^{3,4,5}$. The Outcome Measures in Rheumatology (OMERACT) US Special Interest Group reached a consensus regarding the sonographic elementary lesions that define spondyloarthritis (SpA)-related enthesitis ${ }^{6}$. However, there is no consensus on a global sonographic enthesitis scoring method that can be used to evaluate the extent of enthesitis at the patient level. The GRAPPA US Working Group aims to optimize the evaluation of enthesitis in patients with PsA using US through the development and validation of new instruments by means of a combined data-driven and expert opinion-driven approach. The GRAPPA US Working Group includes GRAPPA members who have expertise in MSK US, methodology experts, and patient research partners from many countries.

\section{GRAPPA US Working Group's Preliminary Work}

The GRAPPA US Working Group's preliminary work highlighted major gaps in knowledge about the validity of

From the University of Toronto and Women's College Hospital, Toronto; University of Ottawa, Ottawa Hospital Research Institute, Ottawa,

Ontario, Canada; University of Florida College of Medicine, Jacksonville, Florida, USA.

As part of the supplement series GRAPPA 2018, this report was reviewed internally and approved by the Guest Editors for integrity, accuracy, and consistency with scientific and ethical standards.

L. Eder, MD, PhD, Assistant Professor of Medicine, University of Toronto and Women's College Hospital; G.S. Kaeley, MD, MBBS, MRCP,

RhMSUS, Professor of Medicine, University of Florida College of

Medicine; S.Z. Aydin, MD, Associate Professor of Medicine, University of

Ottawa, Ottawa Hospital Research Institute.

Address correspondence to Dr. L. Eder, Women's College Hospital,

76 Grenville St., Toronto, Ontario M5S 1B2, Canada.

E-mail:Lihi.EDER@wchospital.ca existing PsA sonographic enthesitis scoring systems. The group's systematic literature review showed that most of the existing scoring methods were developed in patients with predominantly axial SpA (axSpA); the validity of these scoring methods in PsA is unknown ${ }^{7}$. In addition, there is limited information about the discriminative validity and responsiveness of existing PsA scoring systems.

To inform the development of the next steps of the group's project, the group conducted a preliminary study to assess the performance of various sonographic entheseal lesions and sites to distinguish between PsA and healthy controls. The group scanned 50 patients with PsA as well as 50 age- and sex-matched controls. These scans identified 5 potential elemental sonographic lesions and 6 entheseal sites that distinguished PsA from controls ${ }^{8}$. This information will contribute to the development of a research protocol for the development of a new sonographic score for the assessment of enthesitis in patients with PsA.

The group also conducted additional activities, including (1) an online reading exercise that evaluated the group's baseline knowledge of and agreement in the identification of various combinations of sonographic elementary enthesitis lesions, and (2) the acquisition of standard enthesitis images through the electronic submission of images. These activities assisted the group to identify areas of weakness in acquiring and reading images that will be addressed in the next steps of the project.

\section{US Workshop in Toronto, Ontario, Canada}

Following the annual 2018 GRAPPA meeting in Toronto, Ontario, Canada, the GRAPPA US Working Group conducted a 2-day US workshop to discuss the project's next steps. The workshop's aims were to present recent evidence regarding sonographic enthesitis instruments to the members of the working group and to reach a consensus on the research agenda, study design, and US protocols. A total of

Personal non-commercial use only. The Journal of Rheumatology Copyright @ 2019 . All rights reserved. 
33 participants attended the meeting, including rheumatologists who are experts in MSK US, methodology experts, and patient research partners. During the meeting, Dr. Sibel Z. Aydin presented current enthesitis scoring methods, Dr. Maria Antonietta D'Agostino discussed the plans to develop an OMERACT enthesitis scoring system, Dr. Lihi Eder presented summaries of work completed to date by the GRAPPA US Working Group, and Dr. Gurjit S. Kaeley discussed the technical aspects of scanning the enthesis. The meeting also included hands-on scanning of patients with enthesitis to assist with developing a scanning protocol.

The group discussed current unmet needs in the PsA field. With the increased recognition of the importance of early diagnosis and treatment, a diagnostic tool to differentiate psoriasis from PsA is recognized as an important step to improve patient outcomes. There are also previously published scoring methods that are mostly used in ankylosing spondylitis (AS), or axSpA in general, but there is a gap in knowledge about the validity of these tools in the PsA setting ${ }^{7}$. A recent study showed that, despite enthesitis being a frequent manifestation of both AS and PsA, there are differences in the US features. For example, patients with PsA have been shown to have more entheseal damage than patients with $\mathrm{AS}^{9}$. This is in support of the "deep Koebner" phenomenon in PsA that affects sonographic findings despite shared clinical features in both AS and PsA.

Different sonographic scores may be needed to diagnose PsA, to discriminate different disease activity levels, or to assess responsiveness. For example, features of inflammation may be more relevant to discriminate different disease activity levels versus features of damage, whereas abnormal bone proliferation at the entheseal level may have a diagnostic value. Because of this, developing a diagnostic tool that evaluates the constructs of inflammation and damage should be separated from that of developing a disease activity measure that is based on evaluating inflammation. The group agreed its effort should focus on developing an entheseal scoring method for the early diagnosis of PsA at this stage.

The group also agreed to focus on anatomical entheses (e.g., Achilles tendon insertion) and not include functional entheses in the new scoring method at this time. The functional enthesis is seen where there is a change in the direction of muscle pull by wrapping around a bony pulley (e.g., the tibialis posterior wrapping around the medial malleolus). These are sites of high stress where fibrocartilage develops to dissipate the mechanical stress associated with the compression of the tendon against the bone. However, because of the complexity of its assessment and the lack of data on how to evaluate a functional enthesis, the group agreed to restrict the score to the conventional entheses.

Similarly, small entheses, such as the entheses of the hands, are known to be very important in PsA. However, because there has been more work on imaging the large entheses, the group decided to limit the score to the large-to-moderate entheses at this stage. The involvement of the small enthesis is an interesting aspect of the disease that needs to be investigated. However, it first requires more work regarding definitions of sonographic elementary lesions before it can be incorporated in composite score such as the present one.

Another important aspect that needs to be considered is determining which sites should be included in an entheseal scoring method. The group's previous work will inform the working group on the development of a study protocol that validates a new scoring method to ensure that both upper and lower extremity enthesis will be represented in the study.

Central reading, versus the local reading of images collected as part of a future longitudinal study, was also discussed. Local reading has the advantage of performing the scoring during a live scan, which is similar to the real-life situation of using US as a point of care. Conversely, central reading provides more standardization and improves reliability. The group decided to use central reading for the main project, while allowing a smaller group to perform local reading. This will give the group an opportunity to test the agreement between the local and central readings. Cine scans will also be provided in case of pathology to increase the accuracy of the central reading.

It has been well recognized that healthy people may also have features on US at the level of the enthesis. Because the entheses are exposed to biomechanical stress throughout life, there are physiological responses to those mechanical factors that do not necessarily cause any clinical features. It is important to recognize those confounding factors and include them in prospective studies, especially when determining which features are helpful to the diagnosis or assessment of disease activity. A recent study showed that body mass index, sex, age, and physical activity contribute to entheseal abnormalities in healthy people, suggesting that these demographics should be included in the data to be collected ${ }^{10}$. The group agreed that the data collection should also include the disease activity variables of different disease features, such as the skin, entheses, and joints; however, feasibility also needs to be considered.

The group had additional discussions about the technical aspects of scanning the entheses, such as (1) positioning the enthesis to increase sensitivity to detect some of the elementary lesions, (2) scoring abnormalities of the bursa and the tendon in addition to the elementary lesions of enthesitis as defined by the OMERACT group, and (3) defining the machine presets strictly prior to the study versus giving the sonographer some flexibility to change Doppler sensitivity. All of these factors and concerns will be considered when developing the protocol.

\section{Future Steps}

The steering committee is currently working on the protocol to develop a scoring method for diagnostic purposes in PsA

Personal non-commercial use only. The Journal of Rheumatology Copyright $\odot$ 2019. All rights reserved 
that will be shared with GRAPPA members. Those members who are interested, who have the right equipment, and who have sufficient experience will be invited to a training session in 2019. This training session will be followed by an international study in which all these members participate. This study will aim to derive and validate a novel sonographic scoring method that differentiates PsA from psoriasis alone, which could assist in the early diagnosis of PsA. Further, this project will build the foundation for future US studies in psoriasis and PsA by identifying GRAPPA investigators who have expertise in the field.

\section{ACKNOWLEDGMENT}

The authors acknowledge the contribution of the following people who participated in the ultrasound workshop in Toronto and in the activities of the GRAPPA enthesitis working group: Maria Antonietta D'Agostino, Richardo Acayaba, Mariana Alves Ferreira, Catherine Bakewell, Heidi Berthussen, Kristina Callis Duffin, Phillippe Carron, Laura Coates, Joanne Cunha, Diana Flores Alvarado, Dafna D. Gladman, Niti Goel, Marwin Gutierrez, Amir Haddad, Paul Healy, Phillip S. Helliwell, Iustina Janta, Arthur Kavanaugh, Arthur Mandelin, Josefine Marin, Marco Massarotti, Dennis McGonagle, Phillip Mease, Elvia Moreta, Nora Ng, Sarah Ohrndorf, Nirupa Patel, Ari Polachek, Ruben Queiro, Anthony Reginato, Thiele Ralf, Karen Salomon-Escoto, Vibeke Strand, Douglas Veale, Alvin Wells, Michel Yazbek, and Mark Sapsford.

\section{REFERENCES}

1. McGonagle DG, Helliwell P, Veale D. Enthesitis in psoriatic disease. Dermatology 2012;225:100-9.

2. Coates LC, Kavanaugh A, Mease PJ, Soriano ER, Laura Acosta-Felquer M, Armstrong AW, et al. Group for Research and Assessment of Psoriasis and Psoriatic Arthritis 2015 treatment recommendations for psoriatic arthritis. Arthritis Rheumatol 2016;68:1060-71.

3. Kristensen S, Christensen JH, Schmidt EB, Olesen JL, Johansen $\mathrm{MB}$, Arvesen KB, et al. Assessment of enthesitis in patients with psoriatic arthritis using clinical examination and ultrasound. Muscles Ligaments Tendons J 2016;6:241-7.

4. Balint PV, Kane D, Wilson H, McInnes IB, Sturrock RD. Ultrasonography of entheseal insertions in the lower limb in spondyloarthropathy. Ann Rheum Dis 2002;61:905-10.

5. Freeston JE, Coates LC, Nam JL, Moverley AR, Hensor EM, Wakefield RJ, et al. Is there subclinical synovitis in early psoriatic arthritis? A clinical comparison with gray-scale and power Doppler ultrasound. Arthritis Care Res 2014;66:432-9.

6. Terslev L, Naredo E, Iagnocco A, Balint PV, Wakefield RJ, Aegerter $\mathrm{P}$, et al; Outcome Measures in Rheumatology Ultrasound Task Force. Defining enthesitis in spondyloarthritis by ultrasound: results of a Delphi process and of a reliability reading exercise. Arthritis Care Res 2014;66:741-8.

7. Elalouf O, Bakirci Ureyen S, Touma Z, Anderson M, Kaeley GS, Aydin SZ, et al. Psoriatic arthritis sonographic enthesitis instruments: a systematic review of the literature. J Rheumatol 2019;46:43-56.

8. Tom S, Zhong Y, Cook R, Aydin SZ, Kaeley G, Eder L. Development of a preliminary ultrasonographic enthesitis score in psoriatic arthritis - GRAPPA Ultrasound Working Group. J Rheumatol 2018 Oct 15 (E-pub ahead of print).

9. Arslan Alhussain F, Kasapoglu Gunal E, Kurum E, Bakirci S, Ozturk AB, McGonagle D, et al. Greater magnitude of entheseal microdamage and repair in psoriatic arthritis compared with ankylosing spondylitis on ultrasound. Rheumatology 2019; 58:299-303.

10. Solmaz D, Bakirci S, Stephenson W, Eder L, Roth J, Aydin S. The physiological changes of the enthesis in response to age, body mass index and physical activity: an ultrasound study in healthy people [abstract]. Arthritis Rheumatol 2018;70 Suppl 10:1194. 